\title{
MÉTODOS PARA ANÁLISE DE ADAPTABILIDADE E ESTABILIDADE EM CULTIVARES DE TRIGO NO ESTADO DO PARANÁ (1)
}

\author{
LUCIA DE FRANCESCHI $\left({ }^{2}\right)$; GIOVANI BENIN $\left({ }^{*}\right)$; VOLMIR SÉRGIO MARCHIORO $\left({ }^{3}\right)$; \\ THOMAS NEWTON MARTIN $\left({ }^{2}\right)$; RAPHAEL ROSSI SILVA $\left({ }^{2}\right)$; CRISTIANO LEMES DA SILVA $\left({ }^{2}\right)$
}

\begin{abstract}
RESUMO
O presente estudo teve como objetivo avaliar a adaptabilidade e estabilidade para a produção de grãos de 17 cultivares de trigo (Triticum aestivum L.) no Estado do Paraná, utilizando quatro métodos. Os experimentos foram realizados em 2007, em diferentes locais do Paraná, com delineamento blocos casualizados, com quatro repetições. A metodologia de WRICKE (1965) indica cultivares estáveis, independentemente do rendimento médio. Igualmente eficiente na avaliação da estabilidade e indicação de cultivares estáveis e, também adaptadas a ambientes favoráveis e desfavoráveis foram as metodologias de EBERHART e RuSsell (1966) e CRUz et al. (1989). A metodologia de Lin e BINNS (1988), de fácil interpretação, foi eficiente em recomendar cultivares de alto rendimento e boa estabilidade, e os materiais mais responsivos, o menor $P_{i}$ e a alta correlação negativa de Spearman, entre o rendimento de grãos. Concluiu-se que a metodologia de LiN e BinNs é bastante discriminante e, quando associada ao $\mathrm{W}_{\mathrm{i}^{\prime}}$, oferece maior segurança na recomendação de cultivares com maior estabilidade.
\end{abstract}

Palavras-chave: Triticum aestivum L., produção de grãos, adaptabilidade e estabilidade, interação genótipo x ambiente.

\section{ABSTRACT \\ METHODS FOR ANALYSIS OF ADAPTABILITY AND STABILITY OF WHEAT CULTIVARS FOR PARANÁ STATE, BRAZIL}

This investigation had the objective to evaluate grain yield adaptability and stability of 17 wheat cultivars (Triticum aestivum L.) for Paraná State, Brazil, occording to four different methods. The experiments were carried out in 2007, at different locations of Paraná, in complete randomized blocks experimental design, with 4 replicates. The WRICKE (1965) methodology indicates stable cultivars, independently of average yield. EBERHART and RUSSELL (1966) and CRUZ et al. (1989) methodologies were equally efficient to evaluate stability and indicate cultivars that are stable and also adapted to favorable and unfavorable environments. LiN and BINNS (1988) methodology showed to be of easy interpretation and was efficient to indicate cultivars of high yield and with good stability, where more responsive materials showed a lesser $P_{i}$, and high negative correlation of Spearman between grain yields. It was concluded that LIN and BINNS methodology is very specific and when associated to $\mathrm{W}_{\mathrm{i}}$ offers more assurance in recommending cultivars for high stability.

Key words: Triticum aestivum L., grain yield, adaptability and stability, genotype x environment interaction.

(1) Recebido para publicação em 1 de julho de 2009 e aceito em 23 de março de 2010.

$\left({ }^{2}\right)$ Universidade Tecnológica Federal do Paraná - Campus Pato Branco. Via do conhecimento, km 01, 85501-970 Pato Branco (PR). E-mail: luciafranceschi@yahoo.com.br; benin@utfpr.edu.br ( $\left.{ }^{*}\right)$ autor correspondente; martin@utfpr.edu.br; raphaelrossi@agronomo.eng.br; cristianolemes.utfpr@gmail.com

(3) COODETEC, BR 467, Km 98, 85813-450 Cascavel (PR). E-mail: volmir@coodetec.com.br. 


\section{INTRODUÇÃO}

O Estado do Paraná destaca-se como o maior produtor nacional de trigo, contribuindo atualmente com cerca de $60 \%$ da produção nacional do total de 5,04 milhões de toneladas e, próximo de 50\% da área plantada (Companhia..., 2009). Tendo em vista que a produção nacional de trigo representa apenas 50\% do consumo interno, há enorme necessidade em suprir essa lacuna preenchida pela importação. O Paraná possui grande amplitude quanto aos aspectos edafoclimáticos e diferentes riscos climáticos, conferindo grande diversidade no rendimento de trigo (GONÇALVES et al., 1998). Esse fato justifica estudos para identificar genótipos mais adequados às diferentes regiões de cultivo (CAIERÃo et al., 2006), de comportamento previsível, adaptados a ambientes desfavoráveis e que sejam responsivos à melhoria das condições de ambiente (FELICIO et al., 2001; AlbRECHT et al., 2007). Dessa forma, a utilização da análise de adaptabilidade e estabilidade para proceder à recomendação regionalizada, tem sido uma alternativa eficiente e segura.

Diversos métodos são propostos para estimar parâmetros de adaptabilidade e estabilidade, apesar de todos serem fundamentados na interação $G \times A$. Basicamente, esses métodos diferenciam-se quanto aos parâmetros utilizados, na definição de adaptabilidade e estabilidade ou na análise estatística. Apesar de métodos embasados na análise de regressão terem sido os mais utilizados, entretanto, nem todos os dados se ajustam a modelos lineares, o que abre a possibilidade da utilização de outras metodologias como as não-paramétricas e as que se baseiam na análise de regressão bissegmentada (Cruz e Carneiro, 2003).

FinLAY e WiLKINSON (1963) desenvolveram uma metodologia para avaliar o desempenho genotípico para cada genótipo, sendo ajustada uma regressão linear simples da variável dependente em relação ao índice ambiental. Esta difere da metodologia de EBERHART e RusSell (1966), pois estes ampliaram o modelo, considerando também o desvio da regressão para a estimativa da estabilidade. Esse desvio estima a estabilidade de produção, entendida como a previsibilidade do genótipo sob estímulo ambiental. A média da variável dependente fornece um índice ambiental, usado na avaliação do ambiente (CRUZ e CARneiro, 2003).

Na metodologia de VerMA et al. (1978), a cultivar "ideal" é aquela com alta capacidade produtiva e alta estabilidade em ambientes desfavoráveis, mas apta a responderà melhoria doambiente. Pelofato deste método não poder ser aplicado para poucos ambientes, SILVA e BARRETO (1985) aperfeiçoaram essa técnica de forma que os parâmetros de adaptabilidade e de estabilidade passaram a ser estimados pelo ajustamento de uma equação representada por uma reta bissegmentada. Contudo, CRUz et al. (1989) modificaram essa técnica, tornando-a mais simples e com propriedades estatísticas mais adequadas ao melhoramento, possibilitando estimar os parâmetros de adaptabilidade desprovidos de correlações residuais indesejadas.

Ametodologia de WRICKE (1965) identifica a cultivar de desempenho superior, considerando sua média geral, e aquela de comportamento mais previsível, em função das variações temporárias proporcionadas pelo ambiente. Em estudos de comparação de metodologias, DUARTE (1988), concluiu que o método de Wricke, com base em um único parâmetro, é pouco informativo e oferece menor segurança à recomendação de cultivares do que o de análise de regressão de EBERHART e RuSSELl (1966).

Na metodologia proposta por Lin e BINNS (1988), os autores definiram como medida para estimar a estabilidade e adaptabilidade o quadrado médio da distância entre a média da cultivar e a resposta média máxima obtida no ambiente. Essa metodologia foi modificada por CARNEIRO (1998), em que foi sugerida a decomposição do estimador Pi nas partes devidas a ambientes favoráveis e desfavoráveis. Tais modificações trouxeram vantagens ao método como maior facilidade na interpretação, particularização da recomendação das cultivares a grupos de ambientes favoráveis e desfavoráveis. Além disso, contempla o genótipo desejado, já que classifica os genótipos de média alta e constante em ambientes desfavoráveis e com capacidade de resposta à melhoria da condição ambiental, tendo sido eleita por muitos autores a metodologia que melhor classificou e discriminou o conjunto de genótipos (MuraKami et al., 2004; Amorim et al., 2006; Albrecht et al. 2007; CARGNIN et al., 2008).

Estudos de comparações de métodos para avaliar parâmetros de adaptabilidade e estabilidade em trigo são escassos. Portanto, este trabalho tem por objetivo comparar os métodos de ANOVA (WRICKE, 1965), na análise de regressão (EBERHART e RUSSEL, 1966), na análise de regressão bissegmentada (CRUZ et al., 1989) e outro com base na análise não-paramétrica (LiN e BINNS, 1988), em um conjunto de 17 cultivares de trigo avaliado em seis locais do Estado do Paraná.

\section{MATERIAL E MÉTODOS}

Os experimentos foram desenvolvidos no ano agrícola de 2007, em seis regiões edafoclimáticas do Estado do Paraná, ou seja: Cambará, Cascavel, Guarapuava, Londrina, Palotina e Ponta Grossa (Tabela 1). As instituições e órgãos de pesquisa responsáveis pelo manejo dos ensaios foram a Embrapa Soja (Ponta Grossa), o Instituto Agronômico do Paraná - IAPAR (Cambará e Londrina), a Cooperativa Central de Pesquisa Agrícola - 
COODETEC (Cascavel e Palotina) e a Fundação Agrária de Pesquisa Agropecuária - FAPA (Guarapuava). Foram avaliadas 17 cultivares de trigo em sistema de sequeiro e em solo corrigido, ou seja: BRS Guabiju, BRS Camboim, BRS Timbaúva, BRS Louro, BRS Guamirim, BRS 208, BRS 210, CD 104, CD 108, CD 110, CD 111, CD 112, CD 113, CD 114, CD 116, IPR 85 e Ônix (Tabela 2). Os experimentos foram realizados em delineamento de blocos casualizados, com quatro repetições, com a parcela constituída por seis fileiras de $5 \mathrm{~m}$ de comprimento. $\mathrm{O}$ espaçamento entre fileiras foi de 0,2 metros com densidade de semeadura de 350 sementes aptas por $\mathrm{m}^{2}$. A adubação de base e cobertura, assim como o controle de doenças, pragas e plantas daninhas foram realizadas, de acordo com as recomendações técnicas de pesquisa para a cultura (Reunião da Comissão Sul BRASIleira de Pesquisa DE TRIGO, 2007) e as exigências de cada local. A produção de grãos foi transformada $\mathrm{em} \mathrm{kg} \mathrm{ha}^{-1}$ a partir da área útil das parcelas, corrigido a $13 \%$ de umidade.

Paraaanálisedos dados, foi procedida, inicialmente, a verificação das pressuposições do modelo matemático (aleatoriedade dos erros estimados, homogeneidade da variância dos erros estimados entre as cultivares, aditividade do modelo matemático e normalidade da distribuição dos erros estimados) conforme descrições em MARTIN e StORCK (2008). Em seguida procedeu-se às análises de variância individuais para cada experimento visando testar a homogeneidade de variâncias (RAMALHO et al., 2000) e, posteriormente, à análise conjunta dos locais. $\mathrm{Na}$ análise de variância conjunta foram considerados os efeitos dos cultivares fixos e dos ambientes como aleatórios. As comparações de médias foram realizadas pelo teste de SCOTT e KNOTT (1974).

Para a avaliação da adaptabilidade e estabilidade foram utilizados os métodos de WricKe (1965), EBERHART e Russell (1966), Cruz et al. (1989) e de Lin e Binns (1988) com modificações propostas por CARNEIRO (1998). A estatística de estabilidade do método de WRICKE (1965), denominada ecovalência $\left(\varpi_{i}\right)$, foi estimada segundo a equação: $\varpi_{i}=\sum_{j}\left(Y_{i j}-\bar{Y}_{i}-\bar{Y}_{j}+\bar{Y}_{. .}\right)^{2}$. Através desta metodologia, são consideradas estáveis as cultivares com baixos valores de $\varpi_{i^{\prime}}$ o qual indica que estas possuem menores desvios em relação aos ambientes. É uma medida apropriada para expressar a imprevisibilidade do material genético avaliado.

O método de EberHArt e Russell (1966) baseia-se no seguinte modelo de regressãolinear: $Y_{i j}=\mu_{i}+\beta_{i j}+\delta_{i j}+\bar{\varepsilon}_{i j}$. Os coeficientes de regressão de cada cultivar em relação ao índice ambiental $\left(\mathrm{B}_{1}\right)$ e os desvios desta regressão $\left(\delta_{i j}\right)$ proporcionam estimativas de parâmetros de adaptabilidade e estabilidade respectivamente. Para que uma variedade seja considerada "ideal", deve ter, além de uma média elevada, um coeficiente de regressão
Tabela 1. Produtividade de 17 cultivares de trigo cultivadas em seis regiões do Estado do Paraná e a classificação como favoráveis ou desfavoráveis, segundo a metodologia de CRUz et al. (1989)

\begin{tabular}{lcc}
\hline Ambiente & Produtividade & Tipo \\
\hline Cambará & $\mathrm{kg} \mathrm{ha}^{-1}$ & \\
Cascavel & 2568 & Desfavorável \\
Guarapuava & 4206 & Favorável \\
Londrina & 4929 & Favorável \\
Palotina & 4860 & Favorável \\
Ponta Grossa & 3229 & Desfavorável \\
\hline
\end{tabular}

Tabela 2. Genealogia completa das cultivares de trigo avaliadas

\begin{tabular}{lc}
\hline Cultivar & Genealogia \\
\hline CD 104 & PFAU'S”/IAPAR 17 \\
CD 108 & TAM200/TURACO \\
CD 110 & ANAHUAC/PF 869107 \\
CD 111 & PF869107/OCEPAR 18//ANAHUAC \\
CD 112 & IOC 905/PG 877 \\
CD 113 & EMBRAPA 27/OC 946 \\
CD 114 & PF 89232/OC 938 \\
CD 116 & MILAN/MUNIA \\
BRS 208 & CPAC 89118/3/BR 23//CEP 19/PF 85490 \\
BRS 210 & CPAC 89118/3/BR 23//CEP 19/PF 85490 \\
BRS Camboim & Embrapa 27*4/Klein Cartucho//PF 869114/ \\
BRS Guamirim & BR23 \\
BRS Guabiju & Embrapa 27/ Buck Nandu/ PF 93159 \\
BRS Louro & PF 86743/BR 23 \\
BRS Timbauva & PF 869114/BR23 \\
IPR 85 & BR 32/PF 869120 \\
Ônix & IAPAR 30/BR 18 \\
\hline
\end{tabular}

igual ou próximo da unidade e desvios da regressão não diferindo significativamente de zero.

O método de Cruz et al. (1989), baseia-se na análise de regressão linear bissegmentada, sendo expresso da seguinte forma: $Y_{i j}=\beta_{o i}+\beta_{1 i} I_{j}+\beta_{2 i} T\left(I_{j}\right)+\delta_{i j}+\bar{\varepsilon}_{i j}$ Este método considera como cultivar ideal, aquela que tiver média alta (alto $\beta_{o}$ ) adaptabilidade a ambientes desfavoráveis $\left(\beta_{1 i}<1\right)$, responsividade à melhoria ambiental $\left(\beta_{1 i}+\beta_{2 i}\right)>1$ e desvio da regressão zero (não significativo). A estabilidade das cultivares é avaliada pelo desvio da regressão de cada cultivar, em função das variações ambientais. $\mathrm{O}$ índice ambiental empregado pelo método é o mesmo utilizado por EBERHART e RuSSELl (1966), podendo, no entanto ser alternativamente predeterminado em substituição ao índice $I_{j}$. Para cada cultivar é ajustado uma equação de regressão constituída de dois segmentos de reta unidas no ponto correspondente ao valor zero do índice ambiental. Esse método foi ainda utilizado para classificar os ambientes como favoráveis e desfavoráveis para a produção de trigo. 
No método proposto por LiN e BinNs (1988), para estimar a adaptabilidade e a estabilidade, é empregado o quadrado médio da distância entre a média da cultivare a resposta média máxima obtida no ambiente. Essa medida desuperioridadeéobtida por meioda seguinteexpressão:

$P_{i g}=\frac{\sum_{J=1}^{n}\left(X_{i j}-M_{j}\right)^{2}}{2 n}$. O parâmetro estimado pelo método é uma medida relativa a uma cultivar ideal, de adaptabilidade geral, cujo coeficiente de regressão é igual, ou próximo, à unidade. Por esse método representar o quadrado médio da distância em relação à resposta máxima em cada local, e não à distância simples, tem propriedade de variância, ponderando de maneira eficiente o comportamento das cultivares ao longo dos ambientes.

Para que a recomendação de determinada cultivar seja realizada tanto para ambientes favoráveis quanto desfavoráveis, isto é, ambientes em que há emprego de alta e baixa tecnologia, foi sugerido por CARNEIRO (1998) a decomposição do estimador $P_{i}$ do método de LiN e BINNS (1988) nas partes devidas a ambientes favoráveis e desfavoráveis em relação à reta bissegmentada. O parâmetro $P_{i}$ foi denominado MAEC (medida de adaptabilidade e estabilidade de comportamento) e se refere ao desempenho e comportamento diante de variações ambientais. Desta forma, para os ambientes favoráveis, com índice ambiental positivo, incluindo o valor zero, o parâmetro MAEC seria dado por: $P_{i f a v}=\frac{\sum_{j=1}^{f}\left(X_{i j}-M_{j}\right)^{2}}{2 f}$. Da mesma forma, para os ambientes desfavoráveis, com índice ambiental negativo, esse parâmetro seria dado por: $P_{\text {idesf }}=\frac{\sum_{j=1}^{d}\left(X_{i j}-M_{j}\right)^{2}}{2 d}$ Logo, o ideal é um material com o menor $P_{i}$ possível e que a maior parte desse valor seja atribuída ao desvio genético.

Para comparar os parâmetros de adaptabilidade e estabilidade obtidos pelos quatro métodos testados, foi realizado um estudo de correlação de Spearman. Os parâmetros utilizados foram: a média geral dos genótipos, o coeficiente de regressão $\left(\beta_{1 i}\right)$, a variância dos desvios da regressão $\left(\hat{\sigma}_{d i}^{2}\right)$, resposta aos ambientes favoráveis $\left(\beta_{1 i}+\beta_{2 i}\right)$, ambientes desfavoráveis $\left(\beta_{1 i}\right)$, ecovalência $\left(\varpi_{i}\right)$ e os valores de $P_{i}$ geral, $P_{i}$ desfavorável e $P_{i}$ favorável.

As análises de adaptabilidade e estabilidade e a correlação foram realizadas através do aplicativo computacional Genes (CRUZ, 2001).

\section{RESULTADOS E DISCUSSÃO}

As pressuposições do modelo matemático (normalidade da distribuição dos erros estimados, homogeneidade da variância dos erros estimados entre as cultivares, aditividade do modelo matemático) da variável rendimento de grãos, não foram rejeitados. Dessa forma, a qualidade dos experimentos foi garantida para corretos testes de hipóteses (STORCK et al., 2006; MARTIN e STORCK, 2008).

Os ambientes foram avaliados conjuntamente e os coeficientes de variação $(\mathrm{CV})$ se situaram abaixo do limite máximo aceitável de 20\% (Portaria n ${ }^{\circ} 294$ - Lei de proteção de cultivares) demonstrando a qualidade dos resultados obtidos. Além disso, a razão entre o maior e o menor valor do quadrado médio do resíduo foi inferior a 7, que, segundo CRUZ e REGAzZI (1997), é o limite que possibilita a análise de forma conjunta dos experimentos.

Houve diferenças significativas $(P<0,01)$ entre as cultivares, ambientes (locais) e para a interação cultivar $\mathrm{x}$ ambiente, indicando que as cultivares possuem desempenho diferenciado diante das variações ambientais, o que dificulta a recomendação de cultivares para a região estudada, sendo o procedimento recomendado para indicar cultivares a análise de adaptabilidade e estabilidade (CRUZ e CASTOLDI, 1991; Amorim et al., 2006; CARgneluti Filho et al., 2007). Estudando o efeito de duas épocas de semeadura em trigo no Estado de Minas Gerais, CARGNIN et al.(2006) observou interação genótipo $x$ ambiente, concluindo que os genótipos tinham diferentes respostas com relação à condição ambiental devido, possivelmente, à tolerância ao calor. Os autores afirmam que o rendimento de grãos foi o mais afetado pelas condições ambientais, sendo a interação, predominantemente, do tipo complexa, o que dificulta a seleção e indicação dos melhores genótipos.

Constatou-se através da metodologia de Wricke (Tabela 3), que as cultivares BRS $210(1,22)$, BRS 208 $(1,28)$, CD $112(1,01)$, Ônix $(0,57)$, CD $116(1,73)$, BRS Louro $(0,35)$ e CD $108(0,71)$ tiveram os menores valores de $\varpi_{i^{\prime}}$ sendo consideradas as mais estáveis. Porém, destes, apenas as quatro primeiras foram classificadas como de superior rendimento de grãos, com produtividade acima de $4.000 \mathrm{~kg} \mathrm{ha}^{-1}$. Constata-se que nem todas as cultivares de maior estabilidade $\left(\right.$ menor $\mathrm{W}_{\mathrm{i}}$ ) são as que obtiveram alto rendimento em grãos, e resultado semelhante também observado por outros autores (OliveIRA et al., 2002, Cargnelutti Filho et al. 2007). Tal metodologia recomenda cultivares estáveis independentemente do rendimento médio e da responsividade a ambientes favoráveis e desfavoráveis, não sendo conveniente sua utilização quando o objetivo é indicar genótipos para condições específicas de ambiente. 


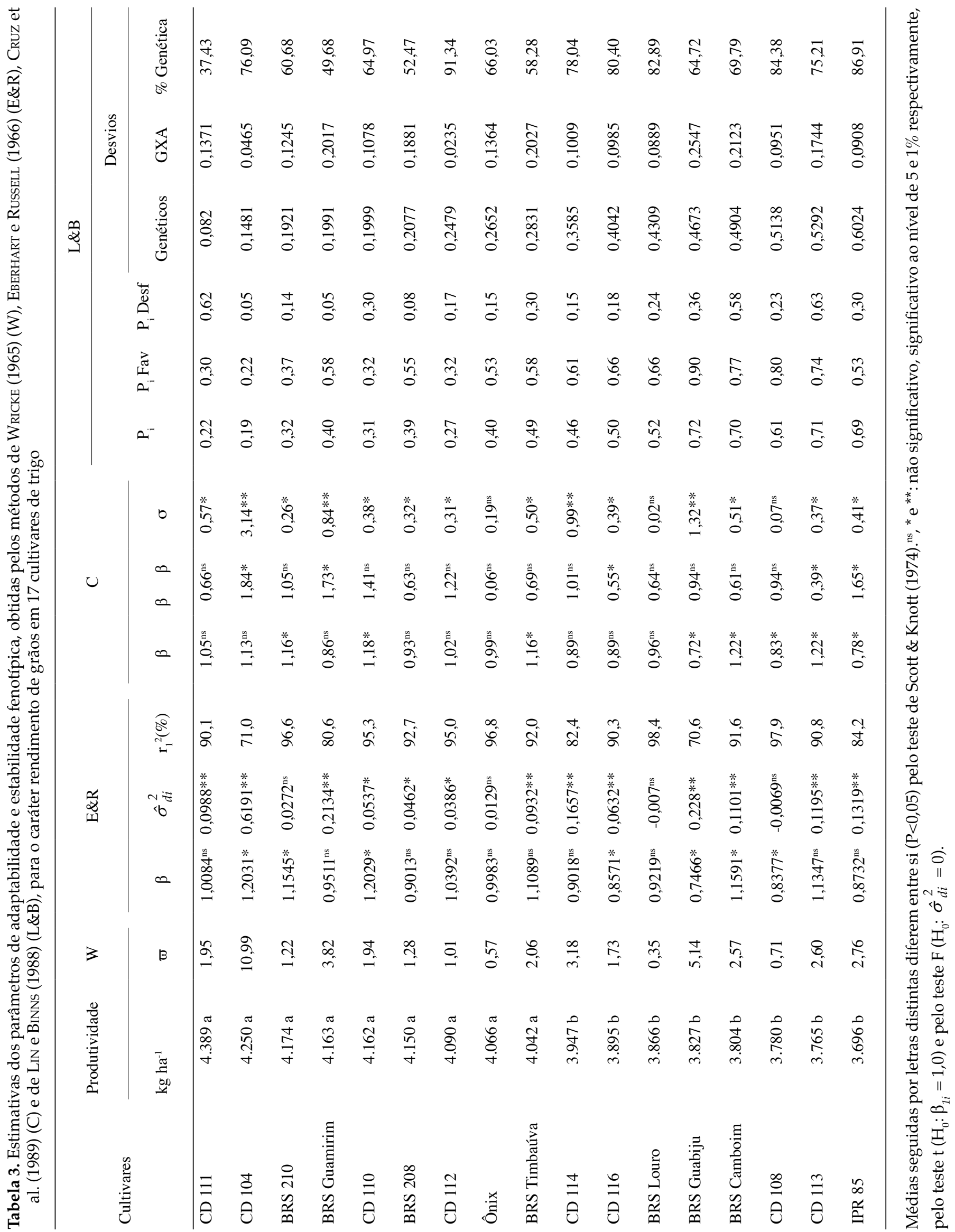


Pela metodologia de EBERHART e Russell (1966), apenas nas cultivares Ônix $\left(\hat{\sigma}_{d i}^{2}=0\right), \mathrm{CD} 111\left(\mathrm{r}^{2}=90,1\right)$, BRS GUAMIRIM $\left(\mathrm{r}^{2}=80,6\right)$, BRS $208\left(\mathrm{r}^{2}=92,7\right), \mathrm{CD} 112$ $\left(r^{2}=95\right)$ e TIMBAÚVA $\left(r^{2}=92\right)$ houve adaptabilidade geral, sendo apenas a primeira com boa estabilidade. Entretanto, OLIVEIRA et al. (2002) afirmam que quando os genótipos possuírem $\mathrm{r}^{2}$ superior a $80 \%$, é indicativo de boa estabilidade. Ainda, é possível destacar a cultivar BRS $210\left(r^{2}=96,6\right)$ e CD $110\left(r^{2}=95,3\right)$ por serem adaptadas a ambientes favoráveis e de alto rendimento de grãos. Tais resultados demonstram que o potencial de rendimento de grãos de trigo é consideravelmente maximizado à medida que melhoram as condições do ambiente de cultivo, como pode ser observado para os municípios de Cascavel, Guarapuava, Londrina e Ponta Grossa (Tabela 1).

Analisando os resultados da metodologia de CRUZ et al. (1989), observa-se que em nenhuma cultivar o desempenho "ideal" foi o preconizado pelo método, ou seja: média alta (alto $\beta_{o}$ ) adaptabilidade a ambientes desfavoráveis $\left(\beta_{1 i}<1\right)$, responsividade à melhoria ambiental $\left(\beta_{1 i}+\beta_{2 i}\right)>1$ e desvio da regressão zero (não significativo). No entanto, a cultivar BRS Guamirim obteve alto rendimento, estando de acordo com o descrito por SCHEEREN et al. (2007), quando afirmam que essa cultivar possui alto desempenho nos diferentes Estados, sendo no Paraná o local em que ocorreu o maior rendimento. Apesar de não haver nenhuma cultivar contemplada como ideal, pode-se inferir que as cultivares BRS Guamirim e CD 104 podem ser recomendadas para ambientes favoráveis, possuindo alto rendimento e baixa estabilidade.

Através da metodologia de LiN e Binss (1988), modificada por Carneiro, as cultivares CD $104\left(\mathrm{P}_{i}=0,19\right)$, CD $111\left(\mathrm{P}_{i}=0,22\right)$ e CD $112\left(\mathrm{P}_{i}=0,27\right)$ foram as que se destacaram com menor valor de $\mathrm{P}_{i}$ (medida de adaptabilidade e estabilidade), havendo ainda, as maiores contribuições da variação genética para esse valor, ou seja, contribuíram pouco para a interação. Por outro lado, as cultivares BRS Guabiju $\left(\mathrm{P}_{i}=0,72\right), \mathrm{CD} 113\left(\mathrm{P}_{i}=0,71\right)$ e BRS Camboim $\left(\mathrm{P}_{i}=0,70\right)$, foram as de desempenho inferior para adaptabilidade geral. Verificou-se também que dentre as nove cultivares com maior rendimento de grãos, apenas duas foram as que obtiveram menores $\mathrm{P}_{i^{\prime}}$ ou seja, a maior parte das cultivares com desempenho médio superior para rendimento de grãos foram também as de melhor adaptabilidade e estabilidade pelo método de LiN e BinNs (1988). Esse fato pode ser explicado pela forma como é estimada a estatística $P_{i^{\prime}}$ que considera como de maior adaptabilidade e estabilidade (menores $\mathrm{P}_{i}{ }^{\prime}$ s), as cultivares cujas as produtividades, em cada ambiente, estejam mais próximos da máxima produtividade (CRUZe CARNEIRO, 2003). Ainda pelo método de Lin e BinNs (1988), considerando osambientesfavoráveis, merecem destaque, novamente, as cultivares CD $104\left(\mathrm{P}_{i f}=0,22\right)$ e CD111 $\left(\mathrm{P}_{i f}=0,30\right)$, sendo um indicativo da sua responsividade à melhoria das condições de ambiente, enquanto para ambientes desfavoráveis merecem destaque as cultivares CD 104, BRS Guamirim e BRS 208.

Quando se comparam os resultados pelas metodologias de Wricke e Eberhart e Russell, é possível notar que cultivares que possuem $B_{1}=1$ er $^{2}>80 \%$, também são as que possuem boa estabilidade pela metodologia de Wricke, indicando redundância de informação entre ambas as metodologias. Quando comparamos a metodologia de Wricke com a de Lin e Binns, é possível observar que há concordância nas cultivares CD 111, BRS 210 e CD 112 com bom desempenho em qualquer dos dois métodos, tanto pelo parâmetro $P_{i^{\prime}}$ que indica cultivares de maiores rendimentos, quanto pelo $\mathrm{W}_{\mathrm{i}}$, indica as cultivares mais estáveis. Dessa forma, é possível inferir que essas cultivares teriam, associando as metodologias, boa estabilidade (menor $\mathrm{W}_{\mathrm{i}}$ ) e alto rendimento (menor $\mathrm{P}_{\mathrm{i}}$ ). Na comparação entre as metodologias de Wricke com Cruz e a de Linn e Binns, é possível observar que as cultivares CD 104 e BRS Guamirim indicadas pelo menor $P_{i}$ estão relacionadas aos maiores escores de $B_{1}+B_{2^{\prime}}$ indicando que as cultivares possuem responsividade à melhoria das condições ambientais, mas consideradas instáveis por possuir alto escore de $\mathrm{W}_{\mathrm{i}}$ e alto desvio pela metodologia de Cruz. Enfim, as metodologias que estimam a estabilidade separadamente são concordantes entre si de que a cultivar CD 104 é a menos estável.

Nos resultados anotados pelo método de EBERHART e RuSSELL (1966) não há qualquer semelhança aos obtidos pelo método de LiN e BINNS (1988), exceto para a condição de ambientes favoráveis. Entretanto, a discordância entre esses métodos é condizente ao constatado por CARgneluti Filho et al. (2007), pois afirmam que esse é um indicativo de que cultivares com menores índices de $\mathrm{P}_{\mathrm{i}}$ na classificação geral e ambientes favoráveis tem maiores escores de $B_{1}$ (CD 104). Dessa forma, menores índices de $\mathrm{P}_{\mathrm{i}}$ indicam maiores rendimentos e resposta a ambientes favoráveis, mas de baixa estabilidade $\left(\hat{\sigma}_{d i}^{2}<0 ; \mathrm{r}^{2}<80 \%\right)$. Esses resultados discordam dos verificados por CAIERÃO et al. (2006), os quais comparando esses dois métodos para a avaliação da adaptabilidade e estabilidade de rendimento de grãos de trigo, observaram que em ambos os métodos houve resultados semelhantes para a maioria das linhagens avaliadas. Da mesma forma, LÉDO et al. (2005), constataram semelhanças nos resultados de ambos os métodos para a produção de matéria seca de alfafa.

A possibilidade de utilização de um ou mais parâmetros de estabilidade obtidos por diferentes metodologias para a predição do comportamento de uma frente às variações ambientais exige $o$ estabelecimento do nível de associação entre estas 
estimativas. Dependendo do grau de associação, esta pode ser uma medida auxiliar na escolha do parâmetro de estabilidade que resulte no melhor ajuste e em mais informações imprescindíveis na formação do seu conceito de estabilidade (DUARTE e ZiMMERMANN, 1995). A precisão requerida e o tipo de informação desejada, são critérios para a escolha do método que ofereça maior segurança na indicação de cultivares (CRUZ e RegAzZI, 1997). Havendo concordância entre os métodos, em relação aos parâmetros de adaptabilidade e estabilidade, há possibilidade de escolher aquele de simples execução e de fácil interpretação. No entanto, caso haja discordância entre os métodos, a indicação de cultivares passa a depender do método utilizado, havendo necessidade de escolha do método mais eficiente.

Em relaçãoao coeficientedecorrelação deSpearman (Tabela 4), foi observada alta correlação negativa $(-0,929$ e -0,793) entre o rendimento de grãos e o parâmetro $P_{i}, P_{i f}$ e baixa correlação negativa $(-0,266)$ entre o rendimento e $\mathrm{P}_{\mathrm{id}^{\prime}}$ significando que quanto maior é o rendimento menor é o valor de $P_{i}$ nas condições de ambiente gerais e favoráveis. Nas condições desfavoráveis, a baixa correlação é explicada por meio da teoria de LiN e BinNs (1988) quando afirmam que a metodologia seleciona cultivares que possuem rendimento elevado e constante nessas condições, revelando que essa metodologia preconiza altos rendimentos.

Também ocorreu alta correlação entre $\hat{\sigma}_{d i}^{2}$ (Eberhart e Russel) e $\varpi_{i}$ (Tabela 4), indicando que quanto maior o desvio do modelo de regressão, maior é o parâmetro de ecovalência e, como a ecovalência avalia as oscilações de cada cultivar diante das variações ambientais. Sendo a cultivar tanto mais estável quanto menor for o valor deste parâmetro, ou seja, quanto maior for à contribuição da cultivar para a interação, maior será a ecovalência e menos estável (TREvisoli, 1999). Estes dados de correlação concordam com os constados por Oliveira et al. (2006), que utilizando os métodos de Lin e Binns, Eberhart e Russell e Wricke para comparação da avaliação de adaptabilidade e estabilidade das características agronômicas de amendoim de porte rasteiro, verificaram correlação negativa entre a produtividade de vagens e massa de mil grãos e o parâmetro $\mathrm{P}_{i}$. Além disso, correlação positiva entre $\hat{\sigma}_{d i}^{2}$ e $\varpi_{i^{\prime}}$ em que é possível concluir que cultivares com alta estabilidade analisadas pelo método de Eberhart e Russel apresentam resultado semelhante pela metodologia de Wricke. Ainda, houve correlação positiva entre o parâmetro $\sigma$ de Cruz com $\mathrm{W}_{\mathrm{i}}(0,982)$ de Wricke e com $\hat{\sigma}_{d i}^{2}(0,982)$ de Eberhart e Russel, indicando concordância na estimativa de estabilidade de Cruz com outros métodos.

Observou-se baixa correlação negativa entre $\mathrm{P}_{i}$ e $\varpi_{i}$ (Tabela 4), podendo-se inferir que nem sempre altos rendimentos podem estar acompanhados de alta estabilidade produtiva, pois esse resultado mostra que pode ocorrer de menor $\mathrm{P}_{\mathrm{i}}$ estar relacionado à maior valor de $\mathrm{W}_{\mathrm{i}^{\prime}}$ concordando com OliveIRA et al. (2006), que detectaram correlação negativa entre $\mathrm{P}_{i}$ e $\varpi_{i}$ para massa de mil grãos. Outro dado importante, é que as estimativas de $\mathrm{P}_{\text {if }}$ foi discordante com o parâmetro $\mathrm{B}_{1}$ de Eberhart e Russel, em que os maiores valores de $B_{1}$ estão relacionados a menores valores de $P_{\text {if }}(--)$, ou seja, cultivares responsivas a ambientes favoráveis pelo parâmetro $B_{1}$ foram também as mais adequadas para ambientes favoráveis pelo parâmetro $P_{\text {if }}$

Considerando que a metodologia de Lin e Binns é de fácil aplicação e interpretação, pois possibilita maior discernimento entre os materiais, apesar de nem sempre associar maior estabilidade com maior produtividade

Tabela 4. Coeficientes de correlação de Spearman entre a média geral do rendimento de grãos (RG, kg ha-1) de 17 genótipos de trigo e os parâmetros: ecovalência $\left(\varpi_{i}\right)$ de WRICKE (1965), coeficiente de regressão $\left(\beta_{1 i}\right)$, a variância dos desvios da regressão $\left(\hat{\sigma}_{d i}^{2}\right)$ de Eberhart e Russel $(1966)$, resposta aos ambientes desfavoráveis $\left(\beta_{1 i}\right)$, resposta aos ambientes favoráveis $\left(\beta_{1 i}+\beta_{2 i}\right)$ e

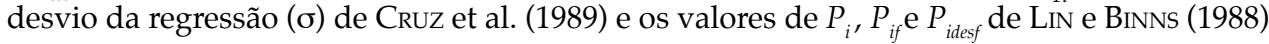

\begin{tabular}{|c|c|c|c|c|c|c|c|c|c|}
\hline & $\mathrm{W}$ & & & & $\mathrm{C}$ & & & LB & \\
\hline & $\varpi_{i}$ & $\beta_{1 i}$ & $\hat{\sigma}_{d i}^{2}$ & $\boldsymbol{\beta}_{1 i}$ & $\beta_{1 i}+\beta_{2 i}$ & $\sigma$ & $P_{i}$ & $P_{i f}$ & $P_{\text {idesf }}$ \\
\hline $\mathrm{RG}$ & $0,188^{\mathrm{ns}}$ & $0,413^{\mathrm{ns}}$ & $0,228^{\mathrm{ns}}$ & $0,324^{\mathrm{ns}}$ & $0,194^{\mathrm{ns}}$ & $0,289^{\mathrm{ns}}$ & $-0,929^{* *}$ & $-0,793^{* *}$ & $-0,266^{\mathrm{ns}}$ \\
\hline$\varpi_{i}$ & & $0,210^{\mathrm{ns}}$ & $0,993^{* *}$ & $0,036^{\text {ns }}$ & $0,572 *$ & $0,982^{* *}$ & $-0,165^{\mathrm{ns}}$ & $-0,215^{\text {ns }}$ & $-0,157^{\mathrm{ns}}$ \\
\hline$\beta_{1 i}$ & & & $0,222^{\mathrm{ns}}$ & $0,947^{* *}$ & $0,112^{\text {ns }}$ & $0,202^{\mathrm{ns}}$ & $-0,408^{\mathrm{ns}}$ & $-0,5614^{*}$ & $0,185^{\mathrm{ns}}$ \\
\hline$\hat{\sigma}_{d i}^{2}$ & & & & $0,449^{\text {ns }}$ & $0,571^{*}$ & $0,982^{* *}$ & $-0,210^{\mathrm{ns}}$ & $-0,257^{\mathrm{ns}}$ & $-0,178^{\mathrm{ns}}$ \\
\hline $\boldsymbol{\beta}_{1 i}$ & & & & & $-0,167^{\mathrm{ns}}$ & $0,035^{\mathrm{ns}}$ & $-0,290^{\mathrm{ns}}$ & $-0,398^{\mathrm{ns}}$ & $0,353^{\mathrm{ns}}$ \\
\hline$\beta_{1 i}+\beta_{2 i}$ & & & & & & $0,528^{*}$ & $-0,276^{\mathrm{ns}}$ & $-0,419^{\text {ns }}$ & $-0,395^{\text {ns }}$ \\
\hline$\sigma$ & & & & & & & $-0,283^{\mathrm{ns}}$ & $-0,284^{\mathrm{ns}}$ & $-0,228^{\mathrm{ns}}$ \\
\hline$P_{i}$ & & & & & & & & $0,892^{* *}$ & $0,432^{\mathrm{ns}}$ \\
\hline$P_{i f}$ & & & & & & & & & $0,272^{\mathrm{ns}}$ \\
\hline
\end{tabular}

ns, * $e^{* *}$ : não significativo, significativo ao nível de 5 e $1 \%$ respectivamente. 
e, por fornecer, maiores informações na indicação das cultivares com adaptabilidade a ambientes favoráveis e desfavoráveis. Assim, sua utilização é bastante vantajosa.

Dessa forma, verifica-se que, de forma generalizada, o parâmetro $W_{i}$ indica cultivares estáveis independentemente do rendimento médio. Já a metodologia de EBERHART e Russel (1966) indica cultivares com maior rendimento, mas associado à adaptabilidade e estabilidade, sendo mais rigoroso no resultado, enquanto o $\mathrm{P}_{\mathrm{i}}$ está associado a altos rendimentos, responsividade às condições favoráveis de ambiente, alto e constante rendimento em condições desfavoráveis, sendo bastante vantajoso o uso da metodologia proposta por LiN e BINNs (1989) pela facilidade de interpretação.

\section{CONCLUSÕES}

1. Onde se deseja alta produtividade, fica evidente a superioridade do estimador $P_{i}$ para a recomendação de cultivares tanto para ambientes favoráveis quanto desfavoráveis, considerando o rendimento de grãos.

2. O método de Lin e Binns é o que melhor discrimina o conjunto de cultivares, em comparação com os métodos de Wricke (1965), Eberhart e Russell (1966) e CRUz et al. (1989), indicando que as cultivares estáveis e responsivas, também são as mais produtivas.

3. O método de Wricke comparado ao de Eberhart e Russel e ao de Cruz indicam forte associação entre si quanto à avaliação de estabilidade, o que torna desnecessário o uso concomitante dessas metodologias.

4. A associação da metodologia de Lin e Binns com Wricke é útil para aumentar a segurança na recomendação de cultivares com alto rendimento de grãos e que sejam estáveis.

\section{REFERÊNCIAS}

ALBRECHT, J.C.; VIEIRA, E.A.; SILVA, M.S.; ANDRADE, J.M.V.A.; SCHEEREN, P.L.; TRINDADE, M.G.; SOBRINHO, J.S.; SOUSA, C.N.A.; REIS, W.P.; JUNIOR, W.Q.R.; FRONZA, V.; CARGNIN, A.; YAMANAKA, C.H. Adaptabilidade e estabilidade de genótipos de trigo irrigado no Cerrado do Brasil Central. Pesquisa Agropecuária Brasileira, v.42, p.17271734, 2007.

AMORIM, E.P.; CAMARGO, C.E.O.; FERREIRA FILHO, A.W.P.; PETTINELLI JUNIOR, A.; GALLO, P.B.; AZEVEDO FILHO, J.A. Adaptabilidade e estabilidade de linhagens de trigo no Estado de São Paulo. Bragantia, v.65, p.575-582, 2006.

CAIERÃO, E; SILVA, M.S.; SCHEEREN, P.L.; DEL DUCA, L.J.A.; JUNIOR, A. N.; PIRES, J.L. Análise da adaptabilidade e da estabilidade de genótipos de trigo como ferramenta auxiliar na recomendação de novas cultivares. Ciência Rural, v.36, p.1112-1117, 2006.

CARGNELUTI FILHO, A.; PERECIN, D.; MALHEIROS, E.B.; GUADAGNIN, J. P. Comparação de métodos de adaptabilidade e estabilidade relacionados à produtividade de grãos de cultivares de milho. Bragantia, v.66, p.571-578, 2007.

CARGNIN, A.; SOUZA, M.A.; FOGAÇA, C.M. Comparação de métodos de análise de adaptabilidade e estabilidade em trigo. Revista Ceres, v.55, p.243-250, 2008.

CARGNIN, A.; SOUZA, M.A.; CARNEIRO, P.C.S.; SOFIATTI, $\mathrm{V}$. Interação entre genótipos e ambientes e implicações em ganhos com seleção em trigo. Pesquisa Agropecuária Brasileira, v.41, p. 987-993, 2006.

CARNEIRO, P.C.S. Novas metodologias de análise da adaptabilidade e estabilidade de comportamento. 1998. 168f. Tese (Doutorado) - Universidade Federal de Viçosa.

COMPANHIA NACIONAL DE ABASTECIMENTO. Acompanhamento de safra brasileira: grãos, sétimo levantamento, abril/2009. Brasília: Conab, 2009. 42p.

CRUZ, C.D. Programa GENES: versão windows: aplicativo computacional em genética e estatística. Viçosa: UFV, 2001. 648p.

CRUZ, C.D.; CARNEIRO, P.C.S. Modelos biométricos aplicados ao melhoramento genético. Viçosa: UFV, 2003. v.2, 585p.

CRUZ, C.D.; CASTOLDI, F.L.Decomposição da interação genótipos $\mathrm{x}$ ambientes em partes simples e complexa. Revista Ceres, v.38, p.422-430, 1991.

CRUZ, C.D.; REGAZZI, A.J. Modelos biométricos aplicados ao melhoramento genético. 2.ed. Viçosa: UFV, 1997. 390p.

CRUZ, C.D.; TORRES, R.A.; VENCOVSKY, R. An alternative approach to the stability analysis proposed by Silva e Barreto. Revista Brasileira de Genética, v.12, p.567-580, 1989.

DUARTE, J.B. Estudo da adaptabilidade e estabilidade fenotípica em linhagens e cultivares de feijão mulatinho (Phaseolus vulgaris (L.)). 155p. 1988. Tese (Mestrado em melhoramento genetico de plantas) - Universidade Federal de Goiás, Goiânia.

DUARTE, J.B.; ZIMMERMANN, M.J. Correlation among yield stability parameters in common bean. Crop Science, v.35, p.905-912, 1995.

EBERHART, S.A.; RUSSEL, W.A. Stability parameters for comparing varieties. Crop Science, v.6, p. 36-40, 1966.

FELICIO, J.C.; CAMARGO, C.E.O.; GERMANI, R.; GALLO, P.B.; PEREIRA, J.C.V.N.A.; BORTOLETO, N.; PETTINELLI JUNIOR, A. Influência do ambiente no rendimento e na qualidade de grãos de genótipos de trigo com irrigação por aspersão no Estado de São Paulo. Bragantia, v.60, p.111-120, 2001. 
FINLAY, K.W.; WILKINSON, G.N. The analysis of adaptation in a plant breeding programme. Australian Journal of Agricultural Research, v.14, p.742-754, 1963.

GONÇALVES, S.L.; CARAMORI, P.H.; WREGE, M.S.; BRUNETTA, D.; DOTTO, S. Regionalização para épocas de semeadura de trigo no Estado do Paraná. Revista Brasileira de Agrometeorologia, v.6, p.239-248. 1998.

LÉDO, F.J.S. Adaptabilidade e estabilidade de cultivares de alfafa avaliadas em Minas Gerais. Ciência e Agrotecnologia, v.29, p.409-414, 2005.

LIN, C.S.; BINNS, M.R.A superiority measure of cultivar performance for cultivar $x$ location data. Canadian Journal of Plant Science, v.68, p.193-198, 1988.

MARTIN, T.N.; STORCK, L. Análise das pressuposições do modelo matemático em experimentos agrícolas no delineamento blocos ao acaso. In: MARTIN, T.N.; ZIECH, M.F. (Org.). SISTEMAS DE PRODUÇÃO AGROPECUÁRIA, 2., 2008, Curitiba. Anais...Curitiba: UTFPR, 2008, v.1, p.177-196.

MURAKAMI, D.M.; CARDOSO, A.A.; CRUZ, C.D.; BIZÃO, N. Considerações sobre duas metodologias de análise de estabilidade e adaptabilidade. Ciência Rural, v.34, p.71-78, 2004.

OLIVEIRA, E.J; GODOY, E.J.; MORAES, A.R.A.; MARTINS, A.L.M.; PEREIRA, J.C.V.N.A.; BORTOLETTO, N.; KASAI, F.S. Adaptabilidade e estabilidade de amendoim de porte rasteiro. Pesquisa Agropecuária Brasileira, v.41, p.1253-1260, 2006.

OLIVEIRA, J.S.; FERREIRA, R.P.; CRUZ, C.D.; PEREIRA, A.V.; BOTREL, M.A.; PINHO, R.G.; RODRIGUES, J.A.S.; LOPES, F.C.F.; MIRANDA, J.E.C. Adaptabilidade e Estabilidade em Cultivares de Sorgo. Revista Brasileira de Zootecnia, v.31, p.883-889, 2002 (Suplemento)
RAMALHO, M.A.P.; FERREIRA, D.F.; OLIVEIRA, A.C. Experimentação em genética e melhoramento de plantas. Lavras: UFLA, 2000. 326p.

REUNIÃO DA COMISSÃO SUL BRASILEIRA DE PESQUISA DE TRIGO, 38., 2006, Passo Fundo, RS. Recomendações da Comissão Sul Brasileira de Pesquisa de Trigo para 2007. Passo Fundo: Embrapa Trigo, 2007. 74p.

SCHEEREN, P.L.; CAIERÃO, E.; SILVA, M.S.; DUCA, L.J.A.; JUNIOR, A.N.; LINHARES, A.; EICHELBERGER, L. BRS Guamirim: cultivar de trigo da classe pão, precoce e de baixa estatura. Pesquisa Agropecuária Brasileira, v.42, p.293-296, 2007.

SCOTT, A.J.; KNOTT, M.A cluster analysis method for grouping means in the analyses of variance. Biometrics, v.30, p.507-512, 1974.

SILVA, J.G.C.; BARRETO, J.N. Aplicação da regressão linear segmentada em estudos da interação genótipo $x$ ambiente. In: SIMPÓSIO DE ESTATÍSTICA APLICADA A EXPERIMENTAÇÃO AGRÍCOLA. Piracicaba. Resumos. Fundação Cargill, Campinas, p.49-50, 1985.

STORCK, L.; GARCIA, D.C.; LOPES, S.J.; ESTEFANEL, V. Experimentação vegetal. Santa Maria: UFSM, 2006. 198p.

TREVISOLI, S.H.U. Estabilidade fenotipica e potencialidade de progênies obtidas por cruzamentos óctuplos em soja. 1999. 228p. Dissertação (Doutorado) - Escola Superior de Agricultura "Luiz de Queiroz", Universidade de São Paulo, Piracicaba, 1999.

VERMA, M.M.; CHAHAL, G.S.; MURTY, B.R. Limitations of conventional regression analysis: a proposed modification. Theoritical and Applied Genetics v.53, p.89-91, 1978.

WRICKE, G. Zur Berechnung der Ökovalenz bei Sommerweizen und Hafer. Zeitschrift für Pflanzenzüchtung, v.52, p.127-138, 1965. 
